\title{
Isolated ophthalmoparesis in a COVID-19 patient A case report and literature review
}

\author{
Maya Aldeeb ${ }^{1}$, Mohamed Samara ${ }^{2}$, Samreen Oraiby ${ }^{2}$, Faraj Howady ${ }^{3}$, Satya Patro ${ }^{4}$, and \\ Dr. Yahia $\operatorname{Imam}^{5}$ \\ ${ }^{1}$ Hamad Medical Corporation Medical Research Center \\ ${ }^{2}$ Hamad Medical Corporation Department of Medical Education \\ ${ }^{3}$ Hamad medical corporation, department of infectious disease \\ ${ }^{4}$ Weil Cornell medicine in Qatar \\ ${ }^{5}$ Hamad Medical Corporation
}

July 31, 2021

\begin{abstract}
We are reporting a COVID-19 positive patient who developed diplopia and was found to have an isolated abducens palsy. We reviewed the available English literature of cranial mononeuropathy as a possible manifestation of COVID-19 infection.It is a rare presentation of COVID-19.
\end{abstract}

\section{Isolated ophthalmoparesis in a COVID-19 patient}

\section{A Case Report and Literature Review}

Maya Aldeeb MD ${ }^{1}$, Mohammad Samara $\mathrm{MD}^{1}$, Samreen Oraiby $\mathrm{MBBS}^{1}$, Faraj Howady $\mathrm{MD}^{2}$, Satya Patro $\mathrm{MD}^{3}$, Yahia Imam MBBS, FRCP(Edin), MSc, FAAN ${ }^{3,4}$

${ }^{1}$ Department of Medical education Hamad Medical Corporation, Doha, Qatar.

${ }^{2}$ Department of Infectious Disease, Hamad Medical Corporation, Doha, Qatar.

${ }^{3}$ Weil Cornell Medicine in Qatar, Doha, Qatar.

${ }^{4}$ Neurology section, Neuroscience Institute, Hamad Medical Corporation, Doha.

Short Title: COVID and Abducens palsy

*Corresponding Author

Maya Aldeeb, Department of Medical Education

Hamad Medical Corporation, Doha, Qatar

Tel: +97450035145

E-mail: maldeeb@hamad.qa

Abstract

COVID-19 has caused a global pandemic, while it is primarily a respiratory pathogen, reports are emerging of neurological manifestation. We are reporting a COVID-19 positive patient who developed diplopia and was found to have an isolated abducens palsy. We reviewed the available English literature of cranial 
mononeuropathy as a possible manifestation of COVID-19 infection. We found that it is a rare presentation of COVID-19. We suggest that an isolated abducens palsy in association with respiratory symptoms should raise the suspicion of COVID-19 infection, particularly in the current pandemic.

Keywords: COVID-19, Diplopia, Abducens palsy, Neurological manifestations

Abbreviations:

CTV (Computed Tomography with Venography); MRI (Magnetic Resonance Imaging); CSF (Cerebrospinal Fluid) ; PCR (Polymerase Chain Reaction), CVT (Cerebral Venous Thrombosis) ; MERS- CoV (Middle Eastern Respiratory Syndrome Coronavirus) ; SARS- CoV (Severe Acute Respiratory Syndrome Coronavirus)

\section{Introduction:}

Since the outbreak of the coronavirus in early December 2019, in Wuhan, China, the virus has rapidly spread throughout the world, gaining a "Pandemic" status. Since then, studies, case reports, and research papers started to be published ${ }^{3,1}$ linking the virus to a myriad clinical manifestation, involving various systems. While the primary target of the virus is the respiratory system, the neurovascular invasiveness of the virus is demonstrated in humans and animals ${ }^{2,3}$. However, some neurological manifestations in COVID-19 patients are reported but still not well-understood.

Studies have shown that the most common neurological manifestations were nonspecific such as headache, dizziness, and agitation ${ }^{4,5}$. Other manifestations include stroke, seizures, and encephalopathy ${ }^{4,6}$. Cranial nerve involvement in COVID-19 patient apart from anosmia and ageusia is rare r. $^{8,7}$.

We present here a case of isolated ophthamloparesis in a patient with a mild COVID-19 pneumonia and a comprehensive literature review of similar cases published in English from December 2019 to end of March 2021.

Case presentation:

A 48-year-old male, previously healthy, was admitted with vomiting, cough and diarrhea, his chest X-ray showed bilateral lower zone infiltrates (figure A), nasopharyngeal swab was positive for COVID-19.

He also complained of binocular diplopia more pronounced on looking to the left that stared a day after his initial manifestation, he had no loss of smell or taste.

On examination, there was a clear limitation of abduction in the left eye with left gaze. Right, upper and lower gazes were intact, as well as convergence (figure B). Other cranial nerve examination was unremarkable including visual acuity, pupillary reflexes and fundoscopy.

General examination was unremarkable, and patient had vitals and oxygen saturation within normal limits without supplemental oxygen. The patient first underwent Computed Tomography with venography (CTV) and mass lesions with increased intracranial pressure and cerebral venous thrombosis was ruled out.

Magnetic Resonance Imaging (MRI) of the head with contrast was done (figure C \& D), and it showed no evidence of any intracranial mass lesions, nor enhancement involving the nerve or its course. A lumbar puncture was performed and showed the cerebrospinal fluid (CSF) to have a normal opening pressure and was acellular, with negative cultures, normal protein, and glucose level. Tuberculosis acid fast bacilli, polymerase chain reaction $(\mathrm{PCR})$ and culture were negative.

Autoimmune screen was also negative and an HBA1c was $5.2 \%$. The patient was treated with hydroxychloroquine, azithromycin, and ceftriaxone as per our local protocol at that time. He also used an eye cover which helped with his diplopia. By day 10 , his $6^{\text {th }}$ nerve palsy has improved remarkably and was discharged to home isolation and outpatient follow up.

Literature review: 
We searched PubMed and Google Scholar from $1^{\text {st }}$ December 2019 to the end of March 2021, we found six articles with a total of 9 patients with ophthalmoparesis secondary to cranial mononeuropathy as a possible neurological manifestation of COVID-19. We excluded patients critically ill, post-intubation, and Miller Fischer patients. Females were the majority $(6 / 9 ; 66.7 \%)$. The mean age of the patients was $42 \pm 14.1$ years (range 2-71); most of them were previously healthy. Out of nine patients, two had third nerve palsy; and seven had sixth nerve palsy. The onset of ophthalmoparesis varied but usually manifested 3-8 days after the start of respiratory symptoms. MRI was done and was unremarkable in 4 out of 6 patients (similar to our patient), interestingly, 7 patients had mild symptoms such as, fever, shortness of breath, anosmia, ageusia, and cough, whereas 2 patients required supplemental oxygen.

Outcomes were documented in 6 patients who responded favorably to conservative treatment and had resolution of the ophthalmoparesis after 14 days to 6 weeks. The cases are summarized in table (1).

\section{Discussion:}

We are reporting, a case with unilateral, isolated abducens nerve palsy in a patient with no known prior co-morbidities, and a mild COVID-19 pneumonia.

Unilateral abducens palsy is the most common type of isolated ocular motor nerve palsies possibly due its small size and long course ${ }^{1,4}$, this also hold true for post COVID-19 infection. A workup to exclude more serious causes such as increased intracranial pressure, stroke, infectious causes, and cerebral vein thrombosis, with stroke and CVT being more suspected than usual in patients with COVID-19, especially with the increasing reports about the thrombogenic potential of the virus ${ }^{1,5}$. While the neurological manifestations of COVID-19 are still being explored, it seems to be more frequent in severe COVID-19 pneumonia unlike our case which was mild. Furthermore, the acellular, aseptic CSF seen in our case is similar to what Gutierrez et al. ${ }^{1,6}$ has reported although the difference that our patient had no ageusia or anosmia .

Neurological injury has been previously described in SARs-CoV and MERS-CoV patients ${ }^{1,7}$. The mechanism of injury is postulated to be through either widespread inflammation, or direct viral invasion of the neuroepithelium and gaining access the central nervous system through the olfactory nerve and olfactory bulb, (anosmia is present in up to $60 \%$ of the cases) $)^{1,8}$, or piggybacking on retrograde axonal transport through other cranial nerves ${ }^{1,9}$.

\section{Conclusion:}

Post COVID-19 cranial nerve involvement is rare. The $6^{\text {th }}$ nerve is more commonly affected than the others and may attest to the virus neuro-invasiveness or enticement of inflammation. The acute presentation of a patient with cranial nerve involvement with or without respiratory symptoms in the current pandemic should prompt investigations for the underlying cause and testing for COVID-19.

\section{Disclosure Statement}

The authors have no conflicts of interest

Ethical statement:

Photographic consent signed by the patient and is available on request

\section{Acknowledgment:}

We would like to acknowledge the Neurology department and Family Medicine Residency program at Hamad Medical Corporation for their support.

Open Access Funding provided by the Qatar National Library

Coauthors:

1.Maya Aldeeb

Department of Medical education Hamad Medical Corporation, Doha, Qatar. 
maldeeb@hamad.qa

2.Mohamed Samara

Department of Medical education Hamad Medical Corporation, Doha, Qatar.

MSamara@hamad.qa

3. Samreen Oraiby

Department of Medical education Hamad Medical Corporation, Doha, Qatar.

samreen.oraiby@gmail.com

4. Faraj Howady:

Department of Infectious Disease, Hamad Medical Corporation, Doha, Qatar.

FHOWADY@hamad.qa

5. Satya Patro:

Weil Cornell Medicine in Qatar, Doha, Qatar

drsatyanpatro@gmail.com

6. Yahia imam

Weil Cornell Medicine in Qatar, Doha, Qatar

Neurology section, Neuroscience Institute, Hamad Medical Corporation, Doha.

yimam@hamad.qa

References:

1. Huang C, Wang Y, Li X, et al. Clinical features of patients infected with 2019 novel coronavirus in Wuhan, China. Lancet Lond Engl . 2020;395(10223):497-506. doi:10.1016/S0140-6736(20)30183-5

2. Li Y, Bai W, Hashikawa T. The neuroinvasive potential of SARS-CoV2 may play a role in the respiratory failure of COVID-19 patients. J Med Virol . Published online March 11, 2020. doi:10.1002/jmv.25728

3. Leisman DE, Deutschman CS, Legrand M. Facing COVID-19 in the ICU: vascular dysfunction, thrombosis, and dysregulated inflammation. Intensive Care Med . 2020;46(6):1105-1108. doi:10.1007/s00134-02006059-6

4. Mao L, Jin H, Wang M, et al. Neurologic Manifestations of Hospitalized Patients With Coronavirus Disease 2019 in Wuhan, China.JAMA Neurol . 2020;77(6):683-690. doi:10.1001/jamaneurol.2020.1127

5. Helms J, Kremer S, Merdji H, et al. Neurologic Features in Severe SARS-CoV-2 Infection. N Engl J Med . 2020;382(23):2268-2270. doi:10.1056/NEJMc2008597

6. Fotuhi M, Mian A, Meysami S, Raji CA. Neurobiology of COVID-19.J Alzheimers Dis JAD . 2020;76(1):319. doi:10.3233/JAD-200581

7. Vaira LA, Salzano G, Deiana G, De Riu G. Anosmia and Ageusia: Common Findings in COVID-19 Patients. The Laryngoscope . 2020;130(7):1787. doi:10.1002/lary.28692

8. Belghmaidi S, Nassih H, Boutgayout S, et al. Third Cranial Nerve Palsy Presenting with Unilateral Diplopia and Strabismus in a 24-Year-Old Woman with COVID-19. Am J Case Rep. Published online 2020:e925897-e925897.

9. Dinkin M, Gao V, Kahan J, et al. COVID-19 presenting with ophthalmoparesis from cranial nerve palsy. Neurology . 2020;95(5):221-223. doi:10.1212/WNL.0000000000009700 
10. Francis JE. Abducens Palsy and Anosmia Associated with COVID-19: A Case Report. Br Ir Orthopt J . 2021;17(1):8-12. doi:10.22599/bioj.167

11. Falcone MM, Rong AJ, Salazar H, Redick DW, Falcone S, Cavuoto KM. Acute abducens nerve palsy in a patient with the novel coronavirus disease (COVID-19). J Am Assoc Pediatr Ophthalmol Strabismus JAAPOS . 2020;24(4):216-217. doi:10.1016/j.jaapos.2020.06.001

12. de Oliveira MR, Lucena ARVP, Higino TMM, Ventura CV. Oculomotor nerve palsy in an asymptomatic child with COVID-19. J AAPOS Off Publ Am Assoc Pediatr Ophthalmol Strabismus . Published online March 6, 2021. doi:10.1016/j.jaapos.2021.02.001

13. Greer CE, Bhatt JM, Oliveira CA, Dinkin MJ. Isolated Cranial Nerve 6 Palsy in 6 Patients With COVID-19 Infection. J Neuro-Ophthalmol Off J North Am Neuro-Ophthalmol Soc . 2020;40(4):520-522. doi:10.1097/WNO.0000000000001146

14. Chan JW, Albretson J. Causes of isolated recurrent ipsilateral sixth nerve palsies in older adults: a case series and review of the literature. Clin Ophthalmol Auckl NZ . 2015;9:373-377. doi:10.2147/OPTH.S78319

15. Middeldorp S, Coppens M, van Haaps TF, et al. Incidence of venous thromboembolism in hospitalized patients with COVID-19. J Thromb Haemost JTH . 2020;18(8):1995-2002. doi:10.1111/jth.14888

16. Gutierrez-Ortiz C, Mendez-Guerrero A, Rodrigo-Rey S, et al. Miller Fisher syndrome and polyneuritis cranialis in COVID-19.Neurology . 2020;95(5):e601-e605. doi:10.1212/WNL.0000000000009619

17. Zegarra-Valdivia JA, Chino-Vilca BN, Tairo-Cerron T, Munive V, Lastarria-Perez C, Ames-Guerrero RJ. Neurological Components in Coronavirus Induced Disease: A Review of the Literature Related to SARS, MERS, and COVID-19. Neurol Res Int . 2020;2020:e6587875. doi:10.1155/2020/6587875

18. Mermelstein S. Acute anosmia from COVID-19 infection. Pract Neurol . 2020;20(4):343-344. doi:10.1136/practneurol-2020-002583

19. Sepehrinezhad A, Shahbazi A, Negah SS. COVID-19 virus may have neuroinvasive potential and cause neurological complications: a perspective review. J Neurovirol . 2020;26(3):324-329. doi:10.1007/s13365020-00851-2

\section{Hosted file}

Table 1 OPHTHALMOPARESIS. docx available at https://authorea.com/users/377042/articles/532295isolated-ophthalmoparesis-in-a-covid-19-patient-a-case-report-and-literature-review 

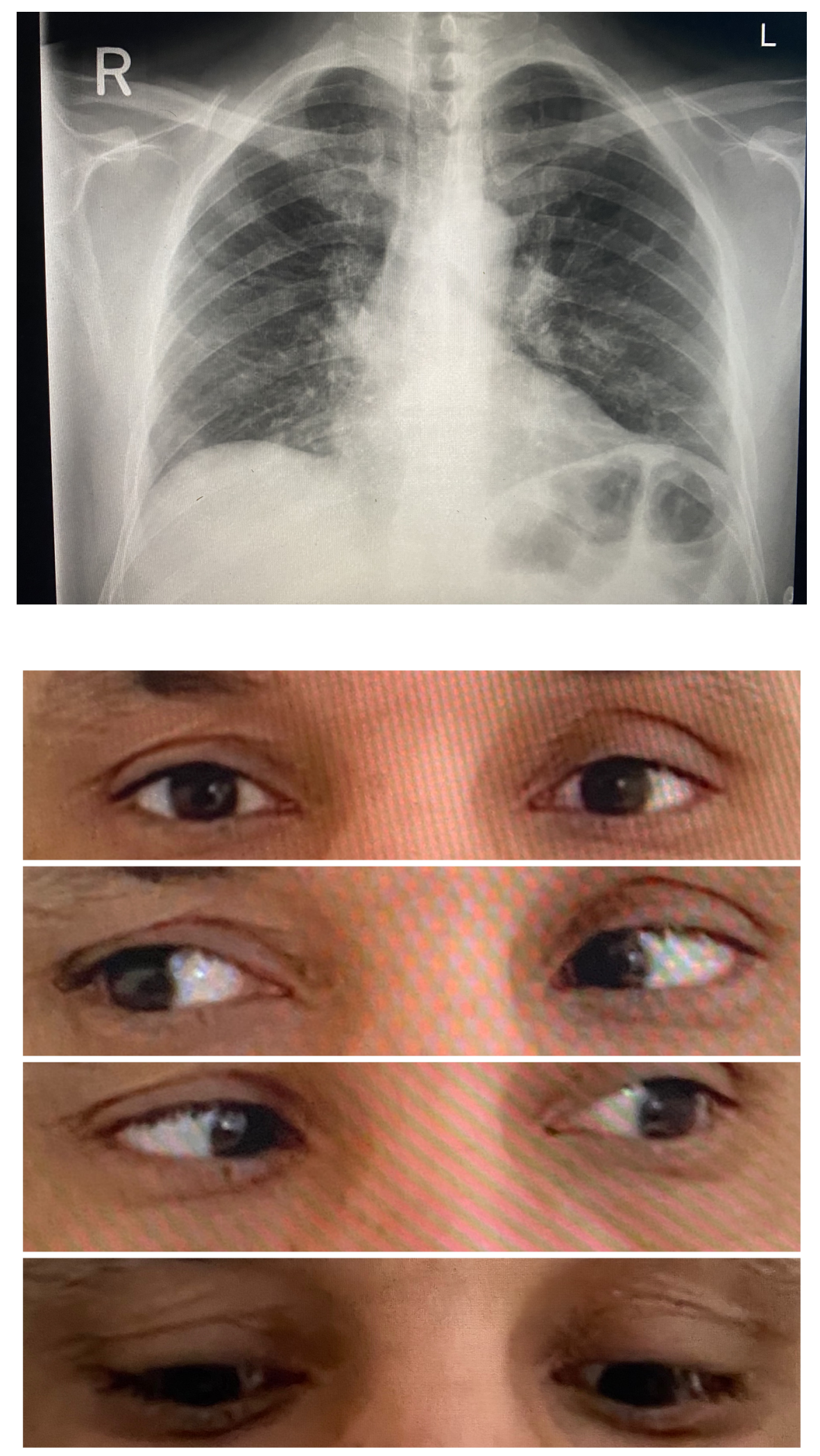

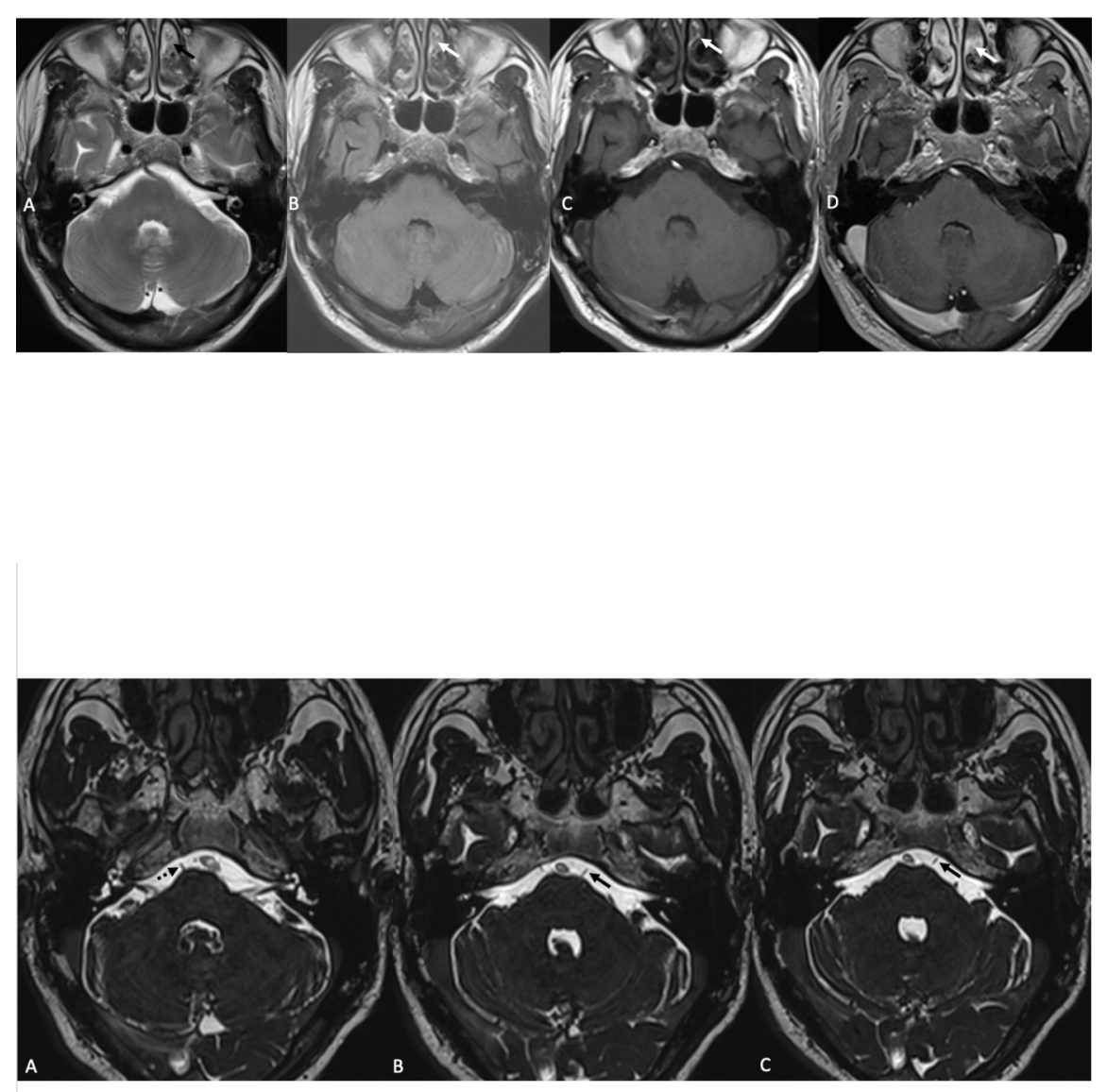